\title{
Spatial interpolation techniques for site-specific irrigation management in a mango orchard
}

\author{
Marcos Sales Rodrigues*, David Castro Alves, Valéria Cristina de Souza, \\ Antônio Carlos de Melo, Augusto Miguel Nascimento Lima, Jailson Cavalcante Cunha
}

Federal University of San Francisco Valley, Petrolina, PE, Brazil

*Corresponding author, e-mail: marcos.rodrigues@univasf.edu.br

\begin{abstract}
Interpolation techniques can be a suitable approach to apply site-specific management for irrigation in fruit fields. However, which interpolation method will produce more accurate maps? In order to respond this question, the aim of this study was to test the performance of spatial interpolation techniques for mapping soil physical properties for site-specific irrigation management purpose in a mango orchard in the Brazilian semi-arid region. The experimental site was structured in a grid of 60 georeferenced points, which correspond to the number of mango trees spaced about $8 \times 10-\mathrm{m}$ in a fruit field in Petrolina county, Pernambuco. Soil texture, bulk density, volumetric water content at $-0.01 ;-0.033 ;-0.06 ;-0.1$; and $-1.5 \mathrm{MPa}$, and soil water available were determined. Stochastic and deterministic interpolation methods were tested. Soil physical properties did not show spatial dependence preventing the interpolation by stochastic method (Kriging). However, it was possible to interpolate using deterministic methods such as inverse distance weight (IDW), local polynomial interpolation (LPI), and Radial basis functions (RBF). IDW and RBF showed the best results of map quality for physical properties, however, all interpolation method showed relative small errors and could be all used for delineating zones for site-specific irrigation management.
\end{abstract}

Keywords: deterministic, Mangifera indica L., precision agriculture, semiarid, stochastic.

\section{Introduction}

The successful cultivation of various fruit species in the semi-arid region of Brazil has been favored by the irrigated agriculture poles. However, in order to provide food for sustainable development, since water resources are limited, agricultural water management must be taken into account (Valipour, 2015). One approach for agricultural water management is site-specific management based on soil physical attributes. Confirming this statement, Nascimento et al. (2014) demonstrate the efficacy of spatial analysis to apply site-specific irrigation management using soil physical properties (i.e, bulk density, soil water retention curve) in a vineyard in the irrigated Petrolina county pole.

The spatial variability of soil properties is often assessed with data interpolation (Silva et al., 2017), however, selecting a proper spatial interpolation method is important, since different methods of interpolation can lead to different results (Li \& Heap, 2011)

Interpolation techniques can be classified into two main groups: deterministic and stochastic interpolation methods. Deterministic interpolation methods use closed-form mathematical formulas or the solution of a linear system of equations to interpolate the data. The weights assigned to each sample value depend only on the distance between the sample point and the location of 
the interpolated point (Varouchakis \& Hristopulos, 2013). The most commonly used deterministic interpolation methods for site-specific agriculture management are inverse distance weighted (IDW) (Li \& Heap, 2011). However, other methods, such as local polynomial interpolation (LPI) and radial basis functions (RBF) have started to be used in agriculture as well (Mueller, 2007, Xie et al., 2011). All these options can be easily performed using most modern GIS (Geographic Information Systems) software packages.

Stochastic methods employ the spatial correlations between values at neighboring points and a semivariogram, which measures the spatial correlation as a function of the distance between data points, should be fitted. The most widely used stochastic method is kriging (Varouchakis \& Hristopulos, 2013).

Different studies have found different results about more suitable interpolation technique (Li \& Heap, 2011), in addition, Robinson \& Metternicht (2006) verified that the best performance of an interpolation technique also depends on the soil variable which is being studied. Since there are no studies testing interpolation technique for delineating irrigation management zones in fruit fields in the semi-arid region of Brazil, the aim of this study is to test the performance of spatial interpolation techniques for mapping soil physical properties for sitespecific irrigation management purpose in a mango orchard in the Brazilian semi-arid region.

\section{Materials and Methods}

This experiment was located in the Nilo Coelho irrigated perimeter, Petrolina County, Pernambuco state, northeastern Brazil (9²1'22.82" S, 40³3'06.95"W, elev. $388 \mathrm{~m}$ a.s.l.). The climate of the region, according to the Köppen classification, is of the 'hot semi-arid' (Bsh') type, characterized by high temperatures (average $26^{\circ} \mathrm{C}$ ), low humidity, high evaporation rates, and especially marked by the scarcity and irregularity in rainfall distribution $(400 \mathrm{~mm})$. The soil of the experimental area was classified as Yellow Argisol (Ultisol - American classification Soil Taxonomy). A 13 years old (2002-2015) commercial mango orchard (cv. Tommy Atkins) irrigated by sprinkler irrigation system was used as the experimental area.

The experimental site was structured in a grid of 60 georeferenced points, which correspond to the number of mango trees spaced about $8 \times 10 \mathrm{~m}$ (Figure 1). Undisturbed soil cores $(0.05 \times 0.05 \mathrm{~m})$ were taken from the 0 to $0.4 \mathrm{~m}$ layer under the canopy of the mango trees in each point using a double-cylinder, hammerdriven core sampler. Additionally, soil disturbed samples of each point were taken from the layer 0 to $0.4 \mathrm{~m}$ using a Dutch auger. Undisturbed soil samples were used to measure soil bulk density (BD) and soil water retention (SWR) at matric potentials $(\psi)$ of 0 and $-0,006$. Sieved $(<2$ $\mathrm{mm}$ ) soil samples were used to measure SWR at $-0.01 ;-0.033 ;-0.06 ;-0.1$; e $-1.5 \mathrm{MPa}$. SWR was determined by the pressure plate apparatus (Soil Moisture Equipment Corp., Santa Barbara, CA), as described by Donagema (2011). For each sample, particle size was determined by the standard pipette method (Donagema, 2011). Soil water available (SWA) was calculated by the following equation (Equation 1)

$$
S W A=\left(\theta_{F C}-\theta_{P P W}\right)
$$

where SWA is the soil water available $\left(\mathrm{cm} \mathrm{cm}^{-3}\right)$, $\theta_{\mathrm{FC}}$ is the volumetric water content $\left(\mathrm{cm} \mathrm{cm}^{-3}\right)$ in the field capacity for sandy soils $(-0.01 \mathrm{MPa})$ and $\theta_{\mathrm{PPW}}$ is the volumetric water content $\left(\mathrm{cm} \mathrm{cm}^{-3}\right)$ in the point of permanent withering (-1.5 MPa).

Descriptive statistical analysis [mean, maximum, and minimum, coefficient of variation (CV), skewness and kurtosis/] were calculated. To test the hypothesis of normality, the Shapiro \& Wilk (1965) test was conducted. According to the method of Warrick \& Nielsen (1980), the variability of soil attributes was classified according to the $\mathrm{CV}$, where a CV value below $15 \%$ was considered low, a value between 15 and $50 \%$ was medium, and $\mathrm{CV}$ greater than $50 \%$ was considered high.

Spatial dependence of samples was tested using semivariogram models (Oliver \& Webster, 2014). However, the study variables showed no spatial dependence or it was very weak (nugget effect value close to sill value), thus, only deterministic interpolation techniques were performed which were: Inverse distance weighting (IDW), Radial basis functions (RBF) and 


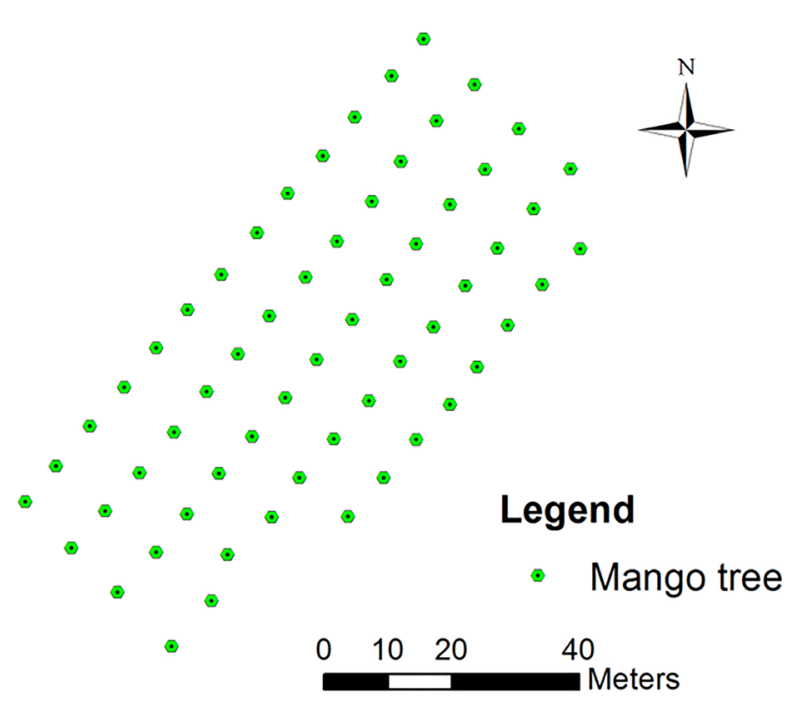

Figure 1. Sampling scheme for soil attributes in an irrigated mango orchard in the semi-arid region, Brazil.

Local Polynomial interpolation (LPI).

IDW interpolation technique is based on the premise that the predictions are a linear combination of available data. Power equal to 2 was used for IDW. RBF is conceptually similar to fitting a rubber membrane through the measured sample values while minimizing the total curvature of the surface. Spline with Tension was the kernel function chosen for RBF. LPI is a process of finding a formula (often a polynomial) whose graph will pass through a given set of points. A complete explanation of these interpolation techniques can be found in Xie et al. (2011).

Cross-validation method was used for comparing the interpolation techniques and indicates which method gives the best results (Sun et al., 2009). This method involves consecutively removing a data point, interpolating the value from the remaining observations and comparing the predicted value with the measured value (Xie et al., 2011). The mean error (ME), mean square error (MSE) and the root mean square error (RMSE) calculated from the measured and interpolated values were used to compare the accuracy of predictions (Li \& Heap, 2011). The ME is defined by (Equation 2):

$$
M E=\frac{1}{n_{v}} \sum_{i=1}^{n_{v}} v_{i}
$$

where $v_{i}$ was the difference between predicted value and observed value at location $s_{i}, i \quad, \ldots$, $n_{v}$, and $n_{v}$ was the number of values in the check data set.
The MSE was the sum of accuracy and precision. It was defined in Equation (3):

$$
M S E=\frac{1}{n_{v}} \sum_{i=1}^{n_{v}} v^{2}{ }_{i}
$$

where $v_{i}^{2}$ was the difference between the square of predicted value and observed value at location $s_{i}, i \quad 1, \ldots, n_{v}$, and $n_{v}$ was the number of values in the check data set. The RMSE was defined as Equation (4) and it represents the error in the variable unit.

$$
R M S E=\sqrt{M S E}
$$

Smaller ME, MSE and RMSE values indicate fewer errors.

\section{Results and Discussion}

Based on the average values of the soil texture, it is verified that the soil is classified as sandy. The minimum and maximum, and coefficient of variation values indicate that there is a high variation of soil texture fractions, mainly for the silt and sand fractions (Table 1). Similar results were found by Rodrigues et al. (2015) in an irrigated guava field in the semi-arid region of Brazil, which verified that even in a small field, soil texture can vary considerably. Therefore, wrong decisions could be made when management of water and fertilizer is defined by average values.

According to Stolf et al. (2011), the soil bulk density limit, which correspond to the BD value when macroporosity (Ma) is equal to $10 \%$ 
(The Ma value of $10 \%$ has been considered a critical limit in relation to soil aeration), can be calculated by its sand content. Therefore, the $\mathrm{BD}$ mean value in the area was below the critical range $\left(1.66 \mathrm{~g} \mathrm{~cm}^{-3}\right)$ in this soil. However, the maximum values may indicate that there are values greater than the $\mathrm{BD}$ limit in the area and problems with soil aeration and drainage may be occurring. According to Warrick \& Nielsen (1980) classification, based on CV values, the water content at all matric potentials and the soil water available (SWA) showed medium variability (CV between 15 and $50 \%)$.

Table 1. Descriptive statistics for soil attributes at the layer of $0-0.40 \mathrm{~m}$ depth in an irrigated mango orchard in the semi-arid region, Brazil.

\begin{tabular}{|c|c|c|c|c|c|c|c|c|c|}
\hline \multirow{2}{*}{ Variables } & \multirow{2}{*}{ Mean } & \multirow{2}{*}{ Min. } & \multirow{2}{*}{ Max. } & \multicolumn{3}{|c|}{ Coefficients } & \multirow{2}{*}{ W-statistic } & \multirow{2}{*}{$\mathrm{Pr}<\mathrm{W}$} & \multirow{2}{*}{ Normality } \\
\hline & & & & Variation & Skewness & Kurtosis & & & \\
\hline Clay & 83 & 7 & 128 & 26 & -0.58 & 1.95 & 0.96 & 0.04 & Non-normal \\
\hline Sand & 841 & 719 & 893 & 4 & -1.76 & 5.12 & 0.86 & 0.00 & Non-normal \\
\hline Silt & 76 & 36 & 207 & 39 & 2.71 & 9.06 & 0.72 & 0.00 & Non-normal \\
\hline $\mathrm{BD}$ & 1.49 & 1.11 & 1.83 & 10 & -0.11 & 0.02 & 0.99 & 0.95 & Normal \\
\hline$\theta 0$ & 0.41 & 0.30 & 0.51 & 12 & -0.57 & -0.05 & 0.96 & 0.07 & Normal \\
\hline$\theta 0.006$ & 0.17 & 0.11 & 0.28 & 22 & 1.19 & 1.10 & 0.89 & 0.00 & Non-normal \\
\hline$\theta 0.01$ & 0.12 & 0.07 & 0.18 & 18 & 0.39 & 0.26 & 0.99 & 0.74 & Normal \\
\hline$\theta 0.033$ & 0.08 & 0.06 & 0.13 & 18 & 1.33 & 2.76 & 0.91 & 0.00 & Non-normal \\
\hline$\theta 0.06$ & 0.07 & 0.05 & 0.09 & 15 & 0.41 & -0.07 & 0.98 & 0.48 & Normal \\
\hline$\theta 1.5$ & 0.06 & 0.04 & 0.09 & 16 & 0.57 & 0.67 & 0.97 & 0.19 & Normal \\
\hline SWA & 0.06 & 0.03 & 0.11 & 31 & 0.26 & -0.48 & 0.98 & 0.49 & Normal \\
\hline
\end{tabular}

The highest coefficients of skewness and kurtosis values were found for the silt content. These results were expected since in soil texture analysis this fraction is obtained by difference, thus, there is a greater estimated error in it. However, although the sand and clay content values showed smaller skewness and kurtosis values comparing to silt content, the normality test showed non-normality of data for these variables. BD and all volumetric water content showed normal distribution of the data, except for those at $0.006 \mathrm{Mpa}$ and $0.033 \mathrm{Mpa}$ matric potentials. Even though Li \& Heap (2011) have reported that normality of data may affect the performance of spatial interpolation methods, Wu et al. (2006) demonstrated that the quality of maps from normal and non-normal data set was very small, then we have chosen not to normalize the data for interpolating.

The prediction accuracy of soil maps values of cross-validation is summarized in Table 2. Based on the Mean Error (ME), Mean Square Error (MSE) and Root Mean Square Error, the accuracy of soil texture maps showing the following order IDW $>$ RBF $>$ LPI. In addition, the greatest difference between interpolation methods was found for soil texture variables (clay, sand, and silt) comparing to the others variables. However, IDW and RBF showed a very similar performance, while LPI showed the worst results. Similar results were found by Mueller (2007) in a central Kentucky field applying ordinary kriging, IDW, RBF, and LPI interpolation procedures. Mueller (2007) verified that LPI procedures produced maps of unreliable quality, while ordinary kriging, IDW interpolation, and RBF produced similar maps.

Clay content is the most important fraction of the soil texture since it is related to the majority of soil chemical and physical attributes and it can significantly affect irrigation management. Therefore, maps with smaller errors of clay content are preferred to define sitespecific management. In the present study, the RMSE varied from 22.6 to $25.1 \mathrm{~kg} \mathrm{~kg}^{-1}$ (Table 2). RMSE provides a measure of the error size (Li \& Heap, 2011), and it can be considerate small even for the worst interpolation method performance $\left(\mathrm{LPI}=25.1 \mathrm{~kg} \mathrm{~kg}^{-1}\right)$ since this error is not sufficiently large to change irrigation management zone. Sumfleth \& Duttmann (2008) found RMSE values varied from $47.6 \mathrm{~g} \mathrm{~kg}^{-1}, 89.67 \mathrm{~g} \mathrm{~kg}^{-1}$ and $56.28 \mathrm{~g}$ $\mathrm{kg}^{-1}$ for clay, sand and silt content, respectively in irrigated fields in the eastern-central part of Jiangxi Province, China. 
Rodrigues et al. (2018) / Spatial interpolation techniques ...

Table 2. The prediction accuracy of interpolation methods for soil attributes at the layer of $0-0.40 \mathrm{~m}$ depth in an irrigated mango orchard in the semi-arid region, Brazil.

\begin{tabular}{|c|c|c|c|c|c|c|c|c|c|}
\hline \multirow{2}{*}{ Variables } & \multicolumn{3}{|l|}{ IDW } & \multicolumn{3}{|l|}{ RBF } & \multicolumn{3}{|l|}{ LPI } \\
\hline & ME & MSE & RMSE & ME & MSE & RMSE & ME & MSE & RMSE \\
\hline Clay & 0.6426 & 511 & 22.6 & 0.2201 & 524 & 22.9 & -2.4869 & 631 & 25.1 \\
\hline Sand & -1.1086 & 1137 & 33.7 & -0.5615 & 1178 & 34.3 & 0.0989 & 1626 & 40.3 \\
\hline Silt & 0.466 & 1103 & 33.2 & 0.3414 & 1170 & 34.2 & 2.3879 & 1941 & 44.1 \\
\hline $\mathrm{BD}$ & -0.0062 & 0.026 & 0.160 & -0.0021 & 0.026 & 0.163 & 0.0087 & 0.030 & 0.173 \\
\hline$\theta 0$ & 0.0022 & 0.002 & 0.049 & 0.0007 & 0.003 & 0.050 & -0.0039 & 0.003 & 0.055 \\
\hline$\theta 0.006$ & -0.001 & 0.002 & 0.039 & -0.0006 & 0.002 & 0.039 & -0.0014 & 0.002 & 0.044 \\
\hline$\theta 0.01$ & -0.0004 & 0.0004 & 0.021 & -0.0002 & 0.0004 & 0.021 & -0.0001 & 0.0005 & 0.022 \\
\hline$\theta 0.033$ & -0.0007 & 0.0002 & 0.015 & -0.0002 & 0.0002 & 0.015 & 0.0008 & 0.0003 & 0.016 \\
\hline$\theta 0.06$ & -0.0002 & 0.0001 & 0.011 & -0.0000 & 0.0001 & 0.011 & 0.0002 & 0.0002 & 0.013 \\
\hline$\theta 1.5$ & -0.0002 & 0.0001 & 0.010 & 0.0000 & 0.0001 & 0.010 & 0.0001 & 0.0001 & 0.011 \\
\hline SWA & -0.0003 & 0.0003 & 0.017 & -0.0002 & 0.0003 & 0.017 & -0.0003 & 0.0003 & 0.018 \\
\hline
\end{tabular}

The RBF technique showed the smaller ME values among the interpolation methods tested (Table 2) for BD and all volumetric water content. ME is used for determining the degree of bias in the estimates, however, according to Li \& Heap et al. (2011) it should be used cautiously as an indicator of accuracy because negative and positive estimates counteract each other and resultant bias tends to be lower than the actual error. On the other hand, based on the RMSE values, IDW showed a slight improvement compared to RBF for BD and soil water content at $0 \mathrm{MPa}$ matric potential (Table 2). For all other variables, IDW and RBF showed the same performance. LPI showed the worst performance for all variables (Table 2).

As stated by Li \& Heap (2011) in their literature review, in most of the studies comparing interpolation methods in environmental sciences, including soil sciences, stochastic techniques (Simple kriging, ordinary kriging, universal kriging, co-kriging etc.) showed better results than deterministic techniques. This can be confirmed by results found by Yasrebi et al. (2009) who verified that ordinary kriging was the most suitable method for prediction and mapping the spatial distribution of soil chemical properties in a fallow land in Iran comparing to IDW using different numbers of power (from 1-5). Sumfleth \& Duttmann (2008) also found that a stochastic method (regression kriging) was more efficient than a deterministic method (Inverse distance weighted, power equal to 1) for clay, sand and silt content in irrigated fields in the eastern-central part of Jiangxi Province, China.

Better results for stochastic methods are expected since samples with a strong spatial structure were mapped more accurately than samples that have weak spatial structure (Xie et al., 2011). Evidently, when there is strong spatial dependence kriging usually is the best choice since kriging is a linear unbiased prediction with minimum variance. However, in some cases, deterministic interpolation techniques are preferred. For example 1) when there are not spatial dependence and semivariograms cannot be fitted (Lu \& Wong, 2008). This is the case of the present study, thus only deterministic methods were applied; 2) number of samples are not enough, since grid size is a very important factor in order to determine the spatial distribution pattern of soil attributes (Rodrigues et al., 2012), grids not very intensive may not be proper for mapping with kriging. 3) In general, IDW, LPI and RBFs are easy to use, due to fewer input parameters used, which is very important for farmers and agronomists to adopt. In contrast, Kriging is more difficult to use since Kriging interpolation including the following steps: statistic test, spatial structure analysis, and semivariance function fitting and so on (Teegavarapu, 2007; Xie et al., 2011). In addition, the semivariance function fitting is subjective, different researchers may have different results (Xie et al., 2011).

In general, the results of the present study demonstrated that IDW and RBF showed almost the same performance. The choice of which map should be used depending on the purpose. In 
this case, the purpose is to define management zones for irrigation, thus, due to RBF provide a smoother surface (Figure 2, 3 and 4) than IDW it should be preferred, whereas maps interpolated by IDW method generate undesirable concentric areas of the same value around the known points more commonly known as "bullseye" (Zưvala et al., 2016), effects which can difficult to delineate management zones (Figures 2, 3 and 4).
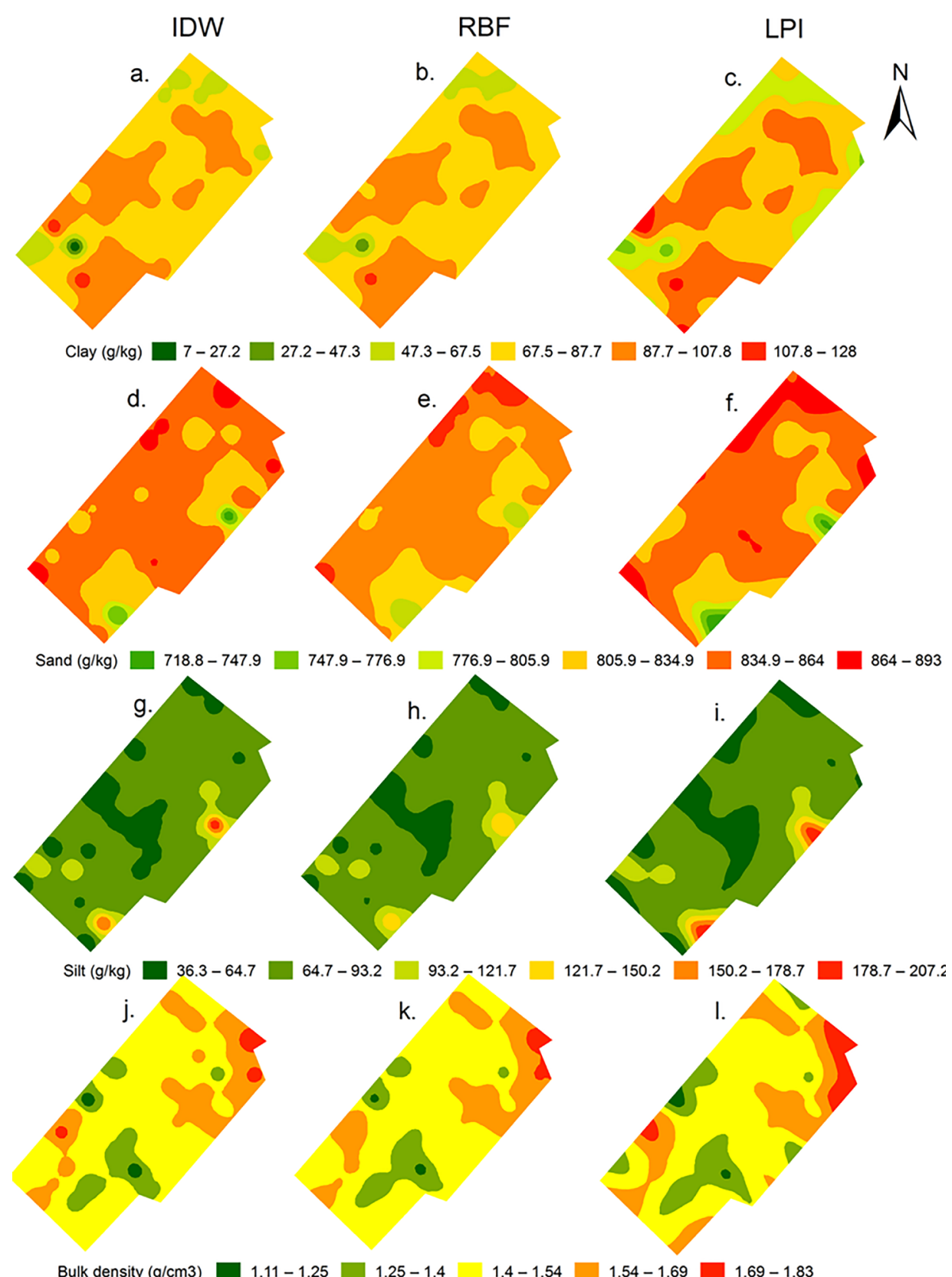

Bulk density $(\mathrm{g} / \mathrm{cm} 3)$

$1.11-1.25 \square 1.25-1.4 \square 1.4-1.54$

$1.54-1.69 \square 1.69-1.83$

$0 \quad 15 \quad 30$ $60 \quad 90$

Figure 2. Clay, Sand, Silt content and Bulk Density maps interpolated by inverse distance weight (IDW), Radial basis functions (RBF) and local polynomial interpolation (LPI) in an irrigated mango orchard in the semi-arid region, Brazil. 

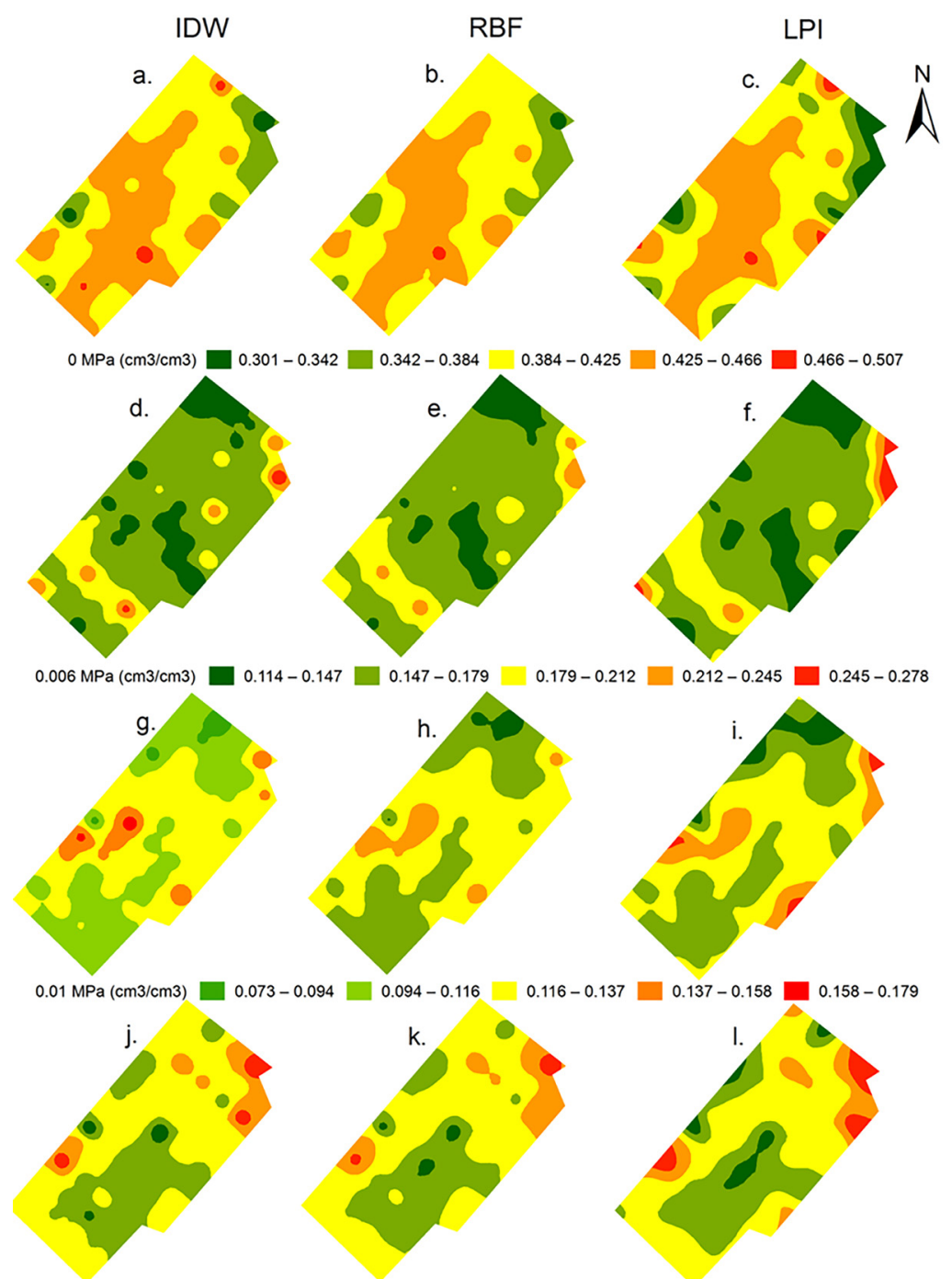

$0.033 \mathrm{MPa}(\mathrm{cm} 3 / \mathrm{cm} 3) \square 0.055-0.068 \square 0.068-0.077 \square 0.077-0.09 \square 0.09-0.107 \square 0.107-0.13$

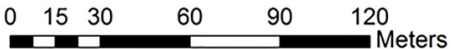

Figure 3. Volumetric water content at $0,0.006,0.01$ and $0.033 \mathrm{MPa}$ tensions maps interpolated by inverse distance weight (IDW), Radial basis functions (RBF) and local polynomial interpolation (LPI) in an irrigated mango orchard in the semi-arid region, Brazil. 

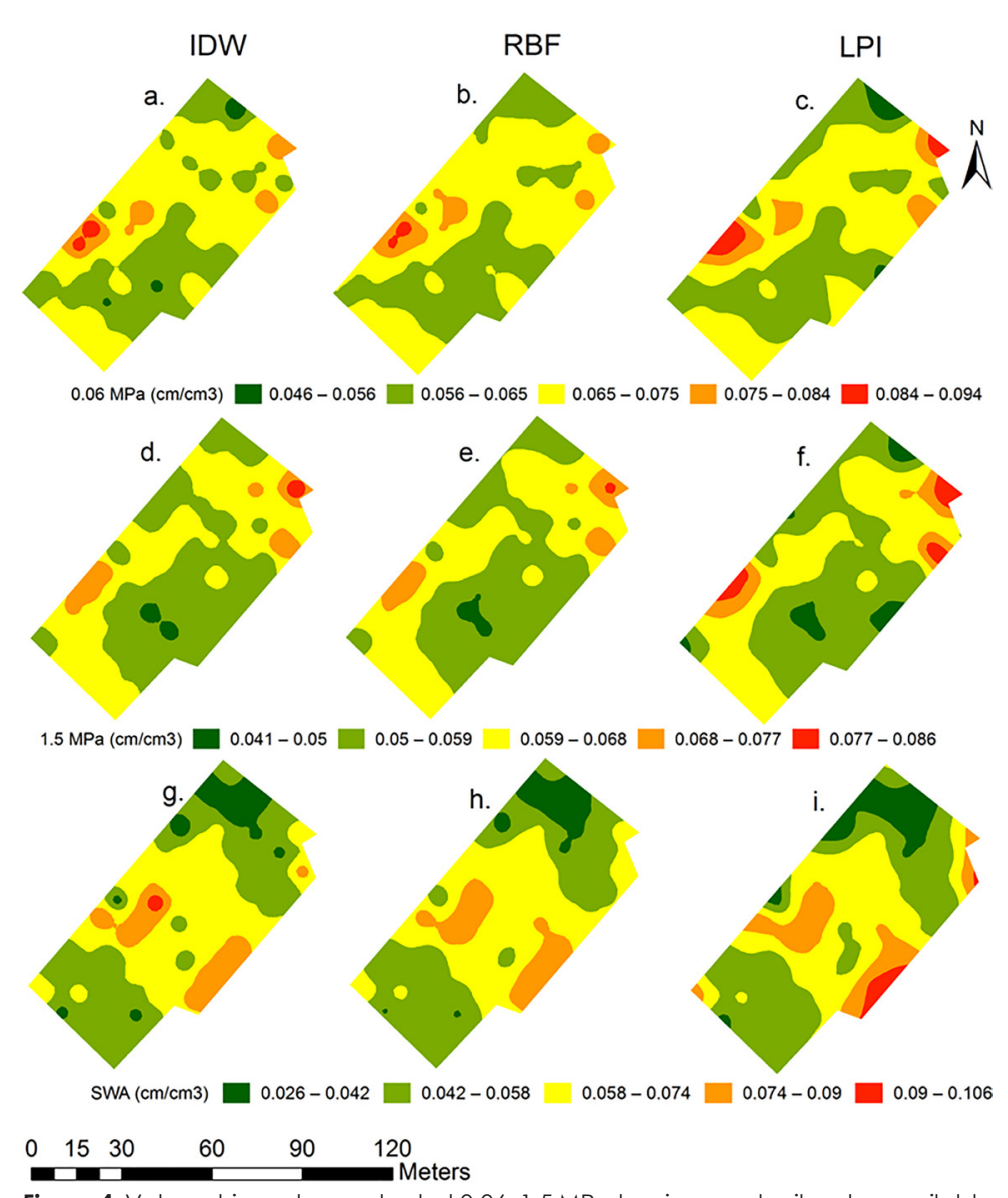

Figure 4. Volumetric water content at 0.06, 1.5 MPa tensions and soil water available (SWA) maps interpolated by inverse distance weight (IDW), Radial basis functions (RBF) and local polynomial interpolation (LPI) in an irrigated mango orchard in the semi-arid region, Brazil.

\section{Conclusions}

The data set of soil physical properties did not show spatial dependence preventing the interpolation by stochastic method (Kriging). However, it was possible to interpolate using deterministic methods such as inverse distance weight (IDW), local polynomial interpolation (LPI) and Radial basis functions (RBF).

IDW and RBF showed the best results of map quality for physical properties in the study area, however, all interpolation method showed relative small errors and can be all used for delineating zones for site-specific irrigation management.

\section{Acknowledgements}

Authors thank to CNPq (National Council for Scientific and Technological Development) for scholarships to the second and the third authors.

\section{References}

Donagema, G.K. 2011. Manual de métodos de análise de solos. Embrapa Solos, Rio de Janeiro, Brazil. $230 \mathrm{p}$.

Li, J., Heap, A.D. 2011. A review of comparative studies of spatial interpolation methods in environmental sciences: Performance and impact factors. Ecological Informatics 6: 228-241.

Lu, G.Y., Wong, D.W. 2008. An adaptive inversedistance weighting spatial interpolation technique. Computers \& Geosciences 34: 10441055.

Mueller, T.G. 2007. Map quality assessment for site-specific fertility management. In: Pierce, F.J., Clay, D. (eds.) GIS Applications in Agriculture. Taylor \& Francis, Boca Raton, USA. p. 103-120.

Nascimento, P.D.S., Silva, J.A., Costa, B.R.S., Bassoi, L.H. 2014. Zonas homogêneas de atributos do solo para o manejo de irrigação em pomar de videira. Revista Brasileira de Ciência do Solo 38: 1101-1113.

Oliver, M.A., Webster, R. 2014. A tutorial guide to geostatistics: Computing and modelling 
variograms and kriging. CATENA 113: 56-69.

Robinson, T.P., Metternicht, G. 2006. Testing the performance of spatial interpolation techniques for mapping soil properties. Computers and Electronics in Agriculture 50: 97-108.

Rodrigues, M.S., Corá, J.E., Fernandes, C. 2012. Soil sampling intensity and spatial distribution pattern of soils attributes and corn yield in notillage system. Engenharia Agrícola 32: 852-865.

Rodrigues, M.S., Santana, M.C., Uchôa, A.L.P., Menezes, A.X.D.S.M.D., Cavalcante, Í.H.L., Lima, A.M.N. 2015. Delineation of management zones based on soil physical attributes in an irrigated guava field in the Semi-Arid region, Brazil. African Journal of Agricultural Research 10: 4185-4192.

Silva, K.A., Rodrigues, M.S., Cunha, J.C., Alves, D.C., Freitas, H.R., Lima, A.M.N. 2017. Levantamento de solos utilizando geoestatística em uma área de experimentação agrícola em Petrolina-PE. Comunicata Scientiae 8: 175-180.

Shapiro, S.S., Wilk, M.B. 1965. An analysis of variance test for normality (complete samples). Biometrika 52: 591-611.

Stolf, R., Thurler, Á.D.M., Bacchi, O.O.S., Reichardt, K. 2011. Method to estimate soil macroporosity and microporosity based on sand content and bulk density. Revista Brasileira de Ciência do Solo 35: 447-459.

Sumfleth, K., Duttmann, R. 2008. Prediction of soil property distribution in paddy soil landscapes using terrain data and satellite information as indicators. Ecological Indicators 8: 485-501.

Sun, Y., Kang, S., Li, F., Zhang, L. 2009. Comparison of interpolation methods for depth to groundwater and its temporal and spatial variations in the Minqin oasis of northwest China. Environmental Modelling \& Software 24: $1163-1170$.

Teegavarapu, R.S.V. 2007. Use of universal function approximation in variance-dependent surface interpolation method: An application in hydrology. Journal of Hydrology 332: 16-29.

Valipour, M. 2015. Assessment of Important Factors for Water Resources Management in European Agriculture. Journal of Water Resource and Hydraulic Engineering 4: 171-180.

Varouchakis, E., Hristopulos, D. 2013. Comparison of stochastic and deterministic methods for mapping groundwater level spatial variability in sparsely monitored basins. Environmental Monitoring and Assessment 185: 1-19.
Warrick, A.W., Nielsen, D.R. 1980. Spatial variability of physical properties in the field. In: Hillel, D. (ed.) Applications of soil physics. Academic Press, New York, USA. p. 319-344.

Wu, J., Norvell, W.A., Welch, R.M. 2006. Kriging on highly skewed data for DTPA-extractable soil Zn with auxiliary information for $\mathrm{pH}$ and organic carbon. Geoderma 134: 187-199.

Xie, Y., Chen, T.-B., Lei, M., Yang, J., Guo, Q.-J., Song, B., Zhou, X.-Y. 2011. Spatial distribution of soil heavy metal pollution estimated by different interpolation methods: Accuracy and uncertainty analysis. Chemosphere 82: 468-476.

Yasrebi, J., Saffari, M., Fathi, H., Karimian, N., Moazallahi, M., Gazni, R. 2009. Evaluation and comparison of ordinary kriging and inverse distance weighting methods for prediction of spatial variability of some soil chemical parameters. Research Journal of Biological Sciences 4: 93-102.

Zưvala, R., Fišerová, E., Marek, L. 2016. Mathematical aspects of the kriging applied on landslide in Halenkovice (Czech Republic). Open Geoscience. 